\title{
Auf neuen Wegen zum EU-Energiemarkt? Ein energiepolitischer Paradigmenwechsel und seine Folgen
}

\author{
Manuel Mohr*
}

Die Europäische Union bemüht sich seit nunmehr 15 Jahren die staatlich protegierten Energiesektoren Europas zu einem funktionierenden europäischen Binnenmarkt für Strom und Gas zu formen. Der Versuch, die ursprünglich rein nationale Marktorganisation der Mitgliedstaaten um eine transnationale, europäische Dimension zu erweitern und einen integrierten Markt zu schaffen, erfolgte dabei jedoch spät. Erst zu Beginn der 1990er Jahre war es der Europäischen Kommission gelungen, den Widerstand einiger nationaler Regierungen, darunter Deutschland und Frankreich, zu überwinden, um auf Grundlage ihrer Zuständigkeit für Angelegenheiten des gemeinsamen Marktes, eine Liberalisierung der europäischen Stromsektoren anzustoßen. ${ }^{1}$ Auf das zugegebenermaßen sehr vorsichtige Aufbrechen bestehender nationaler Strukturen durch die im Jahr 1996 verabschiedete Richtlinie über gemeinsame Vorschriften für den Elektrizitätsbinnenmarkt ${ }^{2}$ folgte 2003 die sogenannte Beschleunigungsrichtlinie. ${ }^{3}$ Diese führte den zuvor begonnenen Prozess der Marktöffnung fort, regulierte den Netzzugang wirkungsvoller und verpflichtete die Mitgliedstaaten zur Einrichtung unabhängiger Regulierungsbehörden. Beide Richtlinien verfolgten damit primär das Ziel, für Wettbewerb auf den Großhandels- und Endkundenmärkten zu sorgen sowie erste Schritte hin zu einer Regulierung der natürlichen Netzmonopole einzuleiten. Anhaltende Wettbewerbsbehinderungen veranlassten die Europäische Kommission jedoch im Herbst 2007 dazu, ein „Drittes Binnenmarktpaket“" vorzulegen, das die europäischen Vorgaben zur gesellschaftsrechtlichen und operativen Entflechtung der Aktivitäten integrierter Energieunternehmen konkretisierte und darüber hinaus das Augenmerk auffallend stark auf Bemühungen zur Marktintegration und Verbesserung der grenzüberschreitenden Zusammenarbeit richtete. Genauer gesagt, spiegelt die neue Gesetzgebung einen Wandel wider, der sich seit einigen Jahren fast unbemerkt in der europäischen Energiepolitik vollzieht. Dieser beruht auf der Einsicht, dass die Vollendung des gemeinsamen Energiebinnenmarktes nur über den Umweg transnationaler regionaler Energiemärkte erreicht werden kann.

Anlässlich des am 3. März 2011 vollständig in Kraft getretenen „Dritten Binnenmarktpakets“, widmet sich der vorliegende Artikel der Erklärung der Energiemarktintegration in Europa unter spezifischer Betrachtung einer pragmatischen Neuausrichtung der Energiepolitik in den letzten Jahren. Die Rede ist von der, zumindest temporären, Abkehr der Europäischen

Manuel Mohr, M.A., Referent für Energie- und Umweltpolitik, Bundesverband der Deutschen Zementindustrie e.V., Berlin.

1 Eising Rainer: Policy Learning in Embedded Negotiations. Explaining EU Electricity Liberalization, in: International Organization 1/2002, S. 88-120.

2 Richtlinie 96/92/EG des Europäischen Parlaments und des Rates vom 19. Dezember 1996 über gemeinsame Vorschriften für den Elektrizitätsbinnenmarkt, in: Amtsblatt der EU, Nr. L 27 vom 19. Dezember 1996, S. 20-29.

3 Richtlinie 2003/54/EG des Europäischen Parlaments und des Rates vom 26. Juni 2003 über gemeinsame Vorschriften für den Elektrizitätsbinnenmarkt, in: Amtsblatt der EU, Nr. L 176 vom 15. Juli 2003, S. 37-55.

4 Richtlinie 2009/72/EG des Europäischen Parlaments und des Rates vom 13. Juli 2009 über gemeinsame Vorschriften für den Elektrizitätsbinnenmarkt und zur Aufhebung der Richtlinie 2003/54/EG; Verordnung (EG) Nr. 714/2009 des Europäischen Parlaments und des Rates vom 13. Juli 2009 über die Netzzugangsbedingungen für den grenzüberschreitenden Stromhandel; Verordnung (EG) Nr. 713/2009 des Europäischen Parlaments und des Rates vom 13. Juli 2009 zur Gründung einer Agentur für die Zusammenarbeit der Energieregulierungsbehörden, alle in: Amtsblatt der EU, Nr. L 211 vom 14. August 2009, S. 1-93. 
Union vom Paradigma des einheitlichen Binnenmarktes zugunsten eines differenzierten regionalen Integrationsansatzes. ${ }^{5}$ Auslöser für diesen unterstellten energiepolitischen Kurswechsel sind die fortbestehende Energiemarktfragmentierung in Europa ebenso wie die Notwendigkeit, den Prozess der Marktintegration an die vorherrschenden Marktrealitäten anzupassen.

Das Bestreben, nationale Strommärkte zunächst auf regionaler Ebene zu harmonisieren und integrieren, erfordert jedoch eine regionale ,market governance', also die Vereinbarung von einheitlichen oder zumindest kompatiblen Standards nationaler Marktorganisation, die den vorherrschenden ,top down'-Ansatz europäischer Sekundärrechtssetzung komplementiert. Regionale Kooperationsstrukturen sind die notwendige Voraussetzung, um die relevanten Marktakteure und Regulierungsbehörden zusammenzubringen und gemeinsam Lösungen für komplexe technische, regulative wie operative Hindernisse der grenzüberschreitenden Marktintegration zu erarbeiten. Sie setzen allerdings die Bereitschaft der genannten Akteure voraus, sich an einer solchen Zusammenarbeit zu beteiligen. Der skizzierte ,bottom up"-Ansatz verfolgt somit eine Strategie der Marktharmonisierung und -integration, die sich parallel zur EU-weiten Regelsetzung, jedoch auf kleinräumigerer Basis vollzieht. Er zeichnet sich ferner durch seinen informellen Charakter und die Tatsache aus, dass jeweils nur Akteure aus einer Gruppe von Mitgliedstaaten regionalspezifische Marktmodelle entwickeln. Zweifellos besteht deshalb die größte Herausforderung dieser Form der, market governance ' in der Sicherstellung einer kohärenten Entwicklung aller Marktregionen, um die angestrebte Vollendung des gemeinsamen Marktes nicht zu gefährden.

Vor diesem Hintergrund geht der vorliegende Artikel zum einen der Frage nach, ob die Energiepolitik der Europäischen Union in den letzten Jahren einen strategischen Richtungswechsel zugunsten eines regionalen Integrationsansatzes vollzogen hat. Zum anderen soll am Beispiel des Stromsektors geklärt werden, ob sich regionale Märkte tatsächlich herausbilden und wenn ja, ob diese der Vollendung des einheitlichen europäischen Energiemarktes Vorschub leisten.

\section{Hindernisse auf dem Weg zum Energiebinnenmarkt}

Wie eingangs angedeutet, haben bis heute alle Bemühungen der Europäischen Union nicht vermocht, einen einheitlichen, nahtlos funktionierenden europäischen Binnenmarkt für den leitungsgebundenen Energieträger Strom zu schaffen. Trotz der in den vergangenen Jahren erzielten Fortschritte in den Bereichen Marktliberalisierung und Netzregulierung stehen der Marktintegration auch heute noch drei zentrale Hindernisse im Weg: 1) Marktkonzentration, 2) die Anwendung des Subsidiaritätsprinzips und 3) mangelnde Leitungsinfrastruktur.

Zunächst offenbart ein Blick auf nationale Marktstrukturen, dass in fast allen 27 Mitgliedstaaten nach wie vor eine geringe Zahl von Unternehmen die nationale Stromversorgung dominiert. Dementsprechend attestierte die Europäische Kommission im letzten Fortschrittsbericht zum Energiebinnenmarkt 20 Mitgliedstaaten weiterhin eine „hohe“ oder sogar ,sehr hohe“ Strommarktkonzentration. ${ }^{6}$ Dieser Umstand ist auf der einen Seite der oft-

5 Für den Zweck der vorliegenden Analyse wird eine Region als transnationale Einheit konzeptualisiert: Sie besteht aus mehreren geografisch benachbarten Mitgliedstaaten sowie den jeweiligen regionalen politischen und wirtschaftlichen Akteuren. Regionale Integration wird hier verstanden als Prozess der Harmonisierung nationaler Marktdesigns und regulativer Regime begleitet von einer infrastrukturellen Vernetzung innerhalb einer Region, mit dem Ziel der Gewährleistung eines unbehinderten grenzüberschreitenden Stromhandels.

6 Insbesondere Belgien, Frankreich, Griechenland, Lettland, Luxemburg und die Slowakei gelten als sehr hoch konzentrierte Erzeugermärkte. Vgl. Europäische Kommission: Mitteilung der Kommission an den Rat und das Europäische Parlament. Bericht über die Fortschritte bei der Verwirklichung des Erdgas- und Elektrizitätsbinnenmarkts, KOM (2010) 84, S. 7; Europäische Kommission: Commission Staff Working Document. Technical Annex to the Communication from the Commission to the Council and the European Parliament: Report on progress in creating the internal gas and electricity market, SEC (2010) 251, S. 12-13. 
mals ineffektiven Umsetzung der EU-Vorgaben zur Entflechtung von Erzeugung und Netzbetrieb geschuldet, wodurch Wettbewerbern integrierter Energieunternehmen der Netzzugang unter anderem durch das Zurückhalten von marktrelevanten Informationen oder die Erhöhung der Kosten für die Netznutzung erschwert wird. ${ }^{7}$ Auf der anderen Seite führt die vertikale Integration von Erzeugung und Endkundenversorgung dazu, dass in einigen Ländern nur begrenzte Liquidität auf den Großhandelsmärkten vorherrscht. Dies erschwert den Marktzugang für neue Erzeuger und unterminiert eine wettbewerbsorientierte Preisbildung. ${ }^{8}$ Nichtsdestotrotz zeigen die Entwicklungen der vergangenen Jahre einen Anstieg des Handelsvolumens auf den Spotmärkten, auf denen Verträge für die kurzfristige Lieferung von Strom geschlossen werden, der in einigen Ländern, wie Italien, Spanien, Griechenland und Nordeuropa bereits mehr als 50 Prozent des nationalen Stromverbrauchs abdeckt. ${ }^{9}$ Auf den Endkundenmärkten der meisten Mitgliedstaaten hingegen kennzeichnet weiterhin träges Wechselverhalten der Konsumenten den Markt und verhindert effektiven Wettbewerb zwischen den Energieversorgern. ${ }^{10}$ Für die Frage der Marktintegration von entscheidender Bedeutung sind jedoch die Auswirkungen oligopolistischer Strukturen auf den grenzüberschreitenden Stromhandel. Ist die Anzahl der Stromerzeuger in einem Mitgliedstaat gering und verfügen diese ebenfalls über Netzinfrastruktur im eigentumsrechtlichen Sinne, haben die Akteure wenig Interesse daran, Konkurrenten aus dem benachbarten Ausland den Zugang zum Markt zu erleichtern. ${ }^{11}$ Dieser Zusammenhang spiegelt sich sowohl im zögerlichen Ausbau von Leitungskapazitäten zwischen benachbarten Staaten wider, also auch in mangelnder Kooperations- und Koordinationsbereitschaft zwischen Netzbetreibern angrenzender Mitgliedstaaten.

Daneben bildet das primärrechtlich verankerte Subsidiaritätsprinzip einen weiteren Stolperstein auf dem Weg zum harmonisierten, gleichsam integrierten Energiemarkt. Wo in vielen Politikbereichen die subsidiäre Verteilung von Verantwortlichkeiten sinnvoll ist, zeigt sich im Fall der Energiemarktregulierung das Gegenteil. Die Umsetzung von EU-weit geltender Rahmengesetzgebung im Geiste der Subsidiarität überlässt den Mitgliedstaaten zwar ausreichend großen Interpretationsspielraum, festigt damit aber die Heterogenität nationaler Strukturen im Energiesektor. ${ }^{12}$ Die subsidiäre Anwendung der weit gefassten europarechtlichen Vorschriften hat so eine Vielzahl nationaler Entflechtungsmodelle und Eigentumsstrukturen, die Einführung unterschiedlicher Regulierungsregime für den Netzbetrieb (Kosten- oder Anreizregulierung) und eine inkonsistente Kompetenzausstattung nationaler Regulierungsbehörden hervorgebracht. Die mangelnde Harmonisierung nationaler Genehmigungsverfahren für den Ausbau grenzüberschreitender Leitungsinfrastruktur ${ }^{13}$ und die

7 Europäische Kommission: DG Competition Report on Energy Sector Inquiry, SEC (2006) 1724, S. 160.

8 Ebenda, S. 157.

9 Europäische Kommission: Commission Staff Working Document, 2010, S. 6, 14.

10 The European Consumers' Association (BEUC): Energy markets in Europe. From dark to light - from cold to heat. The EU Energy Markets after the liberalization: Consumers still waiting to reap the full benefits, Brüssel 2008, S. 11.

11 Jean-Michel Glachant/Francois Lévêque (Hrsg.): Electricity Reform in Europe. Towards a Single Energy Market, Cheltenham/Northampton 2009, hier S. 13; Richard Green: Electricity liberalization in Europe - how competitive will it be?, in: Energy Policy 16/2006, S. 2532- 2541.

12 Roberto J. Serallés: Electric energy restructuring in the European Union: Integration, Subsidiarity and the Challenge of Harmonization, in: Energy Policy 16/2006, S. 2542-2551, hier S. 2546.

13 Jacques De Jong: The "Regional Approach" in establishing the internal EU electricity market. A contribution to regulatory debates in the EU and the US on Standardized Market Designs and Regional Markets. Veröffentlichung des Clingendael International Energy Programme, Den Haag 2004, S. 39; Francois Verneyre: Regional electricity cooperation and integration, in: Janusz Bielecki/Mealku Geboye Desta (Hrsg.): Electricity trade in Europe. Review of the economic and regulatory changes, Den Haag 2004, S. 47-68, hier S. 61. 
Abwesenheit konkreter, bindender europäischer Vorgaben zur Harmonisierung der Großhandelsmärkte, zum Engpassmanagement ${ }^{14}$ und zur Organisation der Regelenergiemärkte ${ }^{15}$ können ferner als Integrationshindernisse angesehen werden. Hierdurch wurde die mangelnde Interoperabilität nationaler Energiesysteme in den vergangenen Jahren weiter gefestigt anstatt diese zu fördern. Das „Dritte Binnenmarktpaket“ stellt in diesem Zusammenhang ein Novum dar, weil die darin enthaltene novellierte Stromhandelsverordnung ${ }^{16}$ nicht nur konkrete Leitlinien zum Engpassmanagement für die gesamte Europäische Union und Vorschriften zur grenzüberschreitenden Koordinierung der Netzbetreiber beinhaltet, sondern auch die Formulierung EU-weit gültiger Netzkodizes für einen diskriminierungsfreien, transparenten und störungsfreien europäischen Netzbetrieb vorsieht. Bedenkt man jedoch, dass bis heute 20 Mitgliedstaaten die Anforderungen der ersten Stromhandelsverordnung ${ }^{17}$ aus dem Jahr 2003 nicht vollständig erfüllen, erscheint das Erreichen einer reibungslosen grenzüberschreitenden Interaktion nationaler Märkte weiterhin als fernes Ziel.

Ein dritter Aspekt, der indirekt mit den beiden vorangegangenen Argumenten verknüpft ist, besteht in der unzureichenden grenzüberschreitenden Leitungsinfrastruktur zwischen den Mitgliedstaaten. Dieser Umstand ist zunächst wenig überraschend, wenn man bedenkt, dass die heutigen Stromnetze einst unter Berücksichtigung rein nationaler Erzeugungs-, Transport- und Versorgungsbedürfnisse installiert wurden. Mit Blick auf die ehrgeizige Zielsetzung der Schaffung eines gemeinsamen europäischen Marktes für Energie verwundert es hingegen, dass bis heute nur ungenügende Anreize zum Infrastrukturausbau geschaffen wurden. ${ }^{18}$ Die begrenzten Transportkapazitäten an vielen Grenzkuppelstellen in Europa offenbaren diesen Missstand und wirken zudem als physisches Hemmnis für einen verstärkten Stromhandel und somit für die Marktintegration. ${ }^{19}$

Die hohe Marktkonzentration gepaart mit der zögerlichen Entflechtung integrierter Energieversorgungsunternehmen stellt eine Ursache für den langsamen Ausbau der Stromnetze in vielen Mitgliedstaaten dar. ${ }^{20}$ Des Weiteren haben sich fehlende Investitionsanreize für eigentumsrechtlich integrierte wie unabhängige Netzbetreiber ebenso wie langwierige, komplexe Genehmigungsverfahren für den (grenzüberschreitenden) Netzausbau in der Vergangenheit als Investitionshemmnis entpuppt. ${ }^{21}$

14 Ein ,,Engpass“ ist eine Situation, in der eine Verbindung zwischen nationalen Übertragungsnetzen wegen unzureichender Kapazität der Verbindungsleitungen und/oder der betreffenden nationalen Übertragungsnetze nicht alle Stromflüsse im Rahmen des von den Marktteilnehmern gewünschten internationalen Handels bewältigen kann." Vgl. Verordnung Nr. 714/2009 über Netzzugangsbedingungen § 2 Abs. 2 lit. c.

15 In Elektrizitätsnetzen muss das Stromangebot zu jeder Zeit des Tages an die schwankende Nachfrage angepasst werden. Regelenergie, die in Primär-, Sekundär- und Tertiärregelung unterteilt wird, ist dabei ,diejenige Energie, die zum Ausgleich von Leistungsungleichgewichten in der jeweiligen Regelzone eingesetzt wird.“ Sie wird auf einem spezifischen Regelenergiemarkt gehandelt. Vgl. § 2 Ziff. 9 Verordnung über den Zugang zu Elektrizitätsversorgungsnetzen (Stromnetzzugangsverordnung - StromNZV) vom 25. Juli 2005 (BGB1. 2005 I, S. 2243-2251).

16 Verordnung (EG) Nr. 714/2009 über Netzzugangsbedingungen.

17 Verordnung (EG) Nr. 1228/2003 des Europäischen Parlaments und des Rates vom 26. Juni 2003 über die Netzzugangsbedingungen für den grenzüberschreitenden Stromhandel, in: Amtsblatt der EU, L 176 vom 15. Juli 2003, S. 1-10.

18 Fabio Domanico: Concentration in the European Electricity Industry: The Internal market as solution?, in: Energy Policy 10/2007, S. 5064-5076, hier S. 5066.

19 Ralph Dickel: Impact of Liberalisation on Investment Performance in the Power Sector, in: Janusz Bielecki/ Melaku Geboye Desta (Hrsg.): Electricity trade in Europe. Review of the economic and regulatory changes, Den Haag 2004, S. 69-94, hier S. 84.

20 Domanico: Concentration in the European Electricity Industry, 2007, S. 5072.

21 Nigel Cornwall: Achieving Electricity Market Integration in Europe, in: Fereidoon Penny Sioshansi (Hrsg.): Competitive Electricity Markets: Design, Implementation, Performance, Amsterdam/London 2008, S. 95-137, hier S. 126. 
Ungeachtet der drei zentralen Hindernisse bei der Schaffung eines einheitlichen europäischen Marktes für Energie haben die von der Europäischen Union angestoßenen Reformen einen Transformationsprozess in Gang gesetzt. So wurde zumindest auf rechtlicher Ebene die Voraussetzung für mehr Wettbewerb auf den Großhandels- und Endkundenmärkten geschaffen. Viel zu lange jedoch hat das Dogma der Marktliberalisierung den Blick für die Anforderungen der Marktintegration verstellt. Die anhaltende Energiemarktfragmentierung ist der beste Beweis dafür. Neben der unausgewogenen energiepolitischen Schwerpunktsetzung der vergangenen Jahre zwischen Marktliberalisierung, also der Schaffung von Wettbewerb in den Bereichen Stromerzeugung (Großhandel) und -lieferung (Endkundenmarkt), der Regulierung des Netzbetriebs sowie der physischen und kommerziellen Integration nationaler Märkte muss dabei auch die bisherige ,market governance“ im Energiebereich infrage gestellt werden. Der lange dominierende ,top down"-Ansatz europäischer Energiemarktreformen sowie dessen subsidiäre Umsetzung haben sich, wie oben erläutert, nicht als zielführend erwiesen. So haben die auf EU-Ebene getroffenen politischen Entscheidungen keine angemessenen Antworten auf die komplexen technischen, regulativen wie wirtschaftlichen Herausforderungen eines integrierten Energiemarktes hervorgebracht. Politische Kompromisse zwischen Europäischer Kommission, Europäischem Parlament und Ministerrat einerseits sowie zwischen den Mitgliedstaaten andererseits genügten zwar, um die Marktbedingungen in den Mitgliedstaaten schrittweise anzugleichen. Für die notwendige Harmonisierung zur Schaffung eines gemeinsamen europäischen Strommarktes hat sich die supranationale Energiepolitik jedoch als untauglich erwiesen. ${ }^{22}$ Daher stellt sich die Frage, wie sich das anvisierte Ziel des Binnenmarktes dennoch realisieren lässt und welche, governance'-Strukturen dies erfordert.

\section{,Bottom up“ versus ,top down' - regionale Integration als Zwischenschritt auf dem Weg zum gemeinsamen Binnenmarkt?}

In der wissenschaftlichen und politischen Debatte werden im Zusammenhang mit einer verbesserten Harmonisierung und Integration nationaler Märkte vorwiegend zwei Handlungsoptionen diskutiert: 1) eine (weitere) Zentralisierung von Kompetenzen und verbindlicher Regelsetzung auf EU-Ebene; 2) die Idee der Dezentralisierung europäischer Marktregulierung und -harmonisierung. Ersteres ist mit der Hoffnung verbunden, einheitlichere Strukturen mittels eines verstärkten ,top down'-Ansatzes, also durch eine intensivere und konkretere Regulierung auf europäischer Ebene, zu erreichen. Allerdings gilt diese Option als politisch schwer umsetzbar, da ein stärkerer Einfluss seitens der Europäischen Union auf die Struktur nationaler Energiemärkte von vielen Mitgliedstaaten nicht gewünscht wird. ${ }^{23}$ Da sich der Erzeugungsmix und die Kraftwerkparks einzelner Mitgliedstaaten bis heute stark voneinander unterscheiden, ist auch aus energiewirtschaftlicher Sicht die Einführung eines standardisierten Marktdesigns für alle Segmente des Strommarktes, wie dies im nordeuropäischen Markt („Nord Pool“) der Fall ist, nicht erstrebenswert. ${ }^{24}$

Die Idee einer Regionalisierung der ,market governance' im Energiesektor, also die zweite Option, hat in der jüngeren Vergangenheit hingegen an Bedeutung gewonnen. Als

22 Tooraj Jamasb/Michael Politt: Electricity Market Reform in the European Union: Review of Progress toward Liberalization \& Integration, Center for Energy and Environment Policy Research, University of Cambridge: Working Paper 05-003, März 2005.

23 Burkhard Eberlein: The Making of the European Energy Market: The Interplay of Governance and Government, in: Journal of Public Policy 1/2008, S. 73-92, hier S. 87.

24 European Federation of Energy Traders: Harmonizing the operation of European Wholesale Electricity Markets, März 2006. 
notwendigen Zwischenschritt zum integrierten Binnenmarkt impliziert sie eine temporäre Übertragung von Verantwortung auf regionale Akteure, um so zunächst regionale Energiemärkte zu formen. ${ }^{25}$ Diese Strategie wird von einigen Energiemarktforschern und inzwischen auch von der Europäischen Kommission als der plausibelste Weg zum Energiebinnenmarkt angesehen, da er die zentralen Akteure einbindet und auf Grundlage bereits existierender Organisationsstrukturen, wie beispielsweise das Florenz Forum ${ }^{26}$ und die European Regulators' Group for Electricity and Gas (ERGEG)“27, umgesetzt werden kann. Nicht zuletzt deshalb, weil dieser , bottom up'-Ansatz die relevanten Marktakteure maßgeblich an der Gestaltung der Regionalmärkte beteiligt, gilt er als der wirtschaftlich sinnvollste Weg zur Vollendung des Binnenmarktes. Doch was spricht für die Schaffung regionaler Energiemärkte und was dagegen?

Diese Frage muss berechtigterweise gestellt werden, da bei kritischer Betrachtung des , bottom up'-Konzepts ein gravierender Nachteil zu Tage tritt. Er impliziert eine temporäre Abkehr vom Paradigma des gemeinsamen Binnenmarktes und birgt damit die Gefahr der Auseinanderentwicklung einzelner Regionen. Um ein späteres Zusammenwachsen der regionalen Märkte zu einem einheitlichen Markt nicht zu gefährden, ist daher eine Koordinierung regionaler ,market governance' sowie die Einhaltung von Mindeststandards in allen Marktregionen essenziell. ${ }^{28}$

Ungeachtet dessen eignet sich der regionale Ansatz jedoch am besten, um die derzeit vorherrschenden Marktrealitäten abzubilden. ${ }^{29}$ Seiner Integrationslogik entsprechend, erlaubt er es zunächst die regionalen Energiemärkte stärker miteinander zu verknüpfen und ihre Marktdesigns zu harmonisieren, die ohnehin schon durch größeren Stromhandel gekennzeichnet sind. Der ,bottom up"-Ansatz kann so schrittweise zur Angleichung nationaler Marktstrukturen beitragen und aus den entstandenen Regionalmärkten im zweiten Schritt einen einheitlichen Binnenmarkt formen. Erfolg verspricht dieser Ansatz allerdings nur unter der bereits genannten Voraussetzung, dass ein Mindestmaß einheitlicher Marktgestaltung und -regulierung in allen Regionen eingehalten wird. Die Regionalen Initiativen sind gerade dabei eine solche Harmonisierung in wichtigen Bereichen herbeizuführen. Mit einheitlicher Marktgestaltung und -regulierung sind die Bereiche gemeint, die zuvor bereits als derzeitige Defizite beschrieben worden sind. Dies erfordert eine wirksame zentrale Koordination sowie eine regelmäßige Überprüfung der regionalen Integrationsfortschritte.

Der regionale Ansatz hat einen weiteren, nicht zu unterschätzenden Vorteil gegenüber der europarechtlichen Regulierung: die geringere Anzahl der Vetospieler, die an der Entscheidungsfindung bei hochkomplexen technischen Fragen, wie der Ausgestaltung des grenzüberschreitenden Stromhandels, beteiligt sind. Im Vergleich zur langwierigen Kompromissfindung unter 27 Mitgliedstaaten mit sehr heterogenen Marktstrukturen trägt die hö-

25 Glachant/Lévêque: Electricity Reform in Europe, 2009; Jamasb/Politt: Electricity Market Reform in the European Union, 2005; De Jong: The "Regional Approach", 2004.

26 Das „European Electricity Regulatory Forum Florence“ kurz Florenz Forum wurde 1998 gegründet und setzt sich aus Vertretern der nationalen Regulierungsbehörden, Netzbetreibern, Stromhändlern, Konsumenten, Energiebörsen sowie aus Mitgliedern nationaler Regierungen und der Europäischen Kommission zusammen. Es tagt ein- bis zweimal pro Jahr und dient als informelles Dialogforum zur europäischen Energiemarktregulierung.

27 Die „European Regulators’ Group for Electricity and Gas (ERGEG)“ wurde im Jahr 2003 von der Europäischen Kommission als formales Beratungsgremium ins Leben gerufen mit dem Auftrag, diese bei der Schaffung eines einheitlichen europäischen Energiebinnenmarktes zu unterstützen. Sie wird Mitte 2011 von der „Agency for the cooperation of energy regulators (ACER)“, die am 3. März 2011 ihre Arbeit aufgenommen hat abgelöst.

28 De Jong: The "Regional Approach", 2004, S. 53.

29 Ebenda, S. 23. 
here Interessenkonvergenz einer kleineren Anzahl von Akteuren in einer Marktregion dazu bei, zunächst spezifisch regionalen Problemen Rechnung zu tragen. Der differenzierte Integrationsansatz weist damit einen wesentlichen Vorteil im Vergleich zu einer, one size fits all'-Politik auf, da er es den beteiligten Marktakteuren und Regulierungsbehörden ermöglicht innerhalb regionaler Strukturen konkretere, den technischen Herausforderungen angemessenere Maßnahmen zu treffen und je nach Harmonisierungsbedarf abweichende Handlungsprioritäten zu setzen. Dadurch werden zunächst die drängendsten Hindernisse für den grenzüberschreitenden Stromhandel in einer Region ausgeräumt, bevor weitere Schritte zur Marktintegration mit benachbarten Regionen erfolgen. Nichtsdestotrotz setzt dieses auf informeller Kooperation basierende Modell voraus, dass die Mehrzahl der beteiligten Akteure ein Interesse an einer Ausweitung des regionalen Stromhandels hat.

\section{,Europe goes regional' - Paradigmenwechsel in der EU-Energiepolitik}

Der skizzierte Modus dezentraler ,market governance‘ auf den europäischen Energiemärkten ist dabei keineswegs ein rein theoretisches Konstrukt. Im Gegenteil hat sich die Energiepolitik der Europäischen Union diesem alternativen, governance'-Modell bereits seit einigen Jahren und auf Drängen der Marktakteure selbst verschrieben. Wenig überraschend hat sich die Einsicht, den einheitlichen Markt in zwei Schritten zu schaffen, zunächst abseits der großen politischen Bühne durchgesetzt. Es ist zu vermuten, dass sich hinter der eher unauffälligen Kursänderung die Angst der Europäischen Kommission verbirgt, der zweistufige Integrationsansatz könnte die grundlegende Idee des einheitlichen Marktes zumindest zeitweise infrage stellen. Die folgende Spurensuche soll daher die Neuausrichtung der europäischen Energiepolitik in den letzten Jahren nachvollziehen, aber zuvor die bestehenden Formen der Energiemarktregulierung in der Europäischen Union kurz darlegen.

Neben dem ordentlichen Gesetzgebungsverfahren gemäß Art. 294 Vertrag über die Arbeitsweise der Europäischen Union (AEUV) ${ }^{30}$ lassen sich im Bereich des Energiebinnenmarktes schon seit geraumer Zeit informelle, governance'-Strukturen im Energiebereich beobachten. Simon Bulmer et al. ${ }^{31}$ zufolge, herrscht in der EU-Energiepolitik bereits seit Längerem eine Form hybrider, governance', die der supranationalen Ebene durch die Zuständigkeit für Angelegenheiten des Binnenmarktes, die Umweltpolitik und transeuropäische Netze zwar einen gewissen Einfluss einräumt, jedoch die letztendliche Marktgestaltung nationalen Akteuren überlässt. Aus diesem Grund, so die Autoren, suchte die Kommission in der Vergangenheit neben der Anwendung des Wettbewerbsrechts, alternative Wege der Einflussnahme auf die Entwicklung der Energiemärkte, um die genannten supranationalen Kompetenzdefizite zumindest teilweise auszugleichen. Neben „,soft“ powers of persuasion“32, wie beispielsweise die regelmäßige Veröffentlichung von Fortschrittsberichten zur Entwicklung des Binnenmarktes und der Entwicklung von Benchmarks für den Energiesektor, regt die Kommission daher die Zusammenarbeit von nationalen Regulierungsbehörden untereinander, aber auch die mit anderen Marktakteuren an, um die Schaffung eines wettbewerbsfähigen Energiebinnenmarktes parallel zum ordentlichen Gesetzgebungsverfahren voranzutreiben.

30 Bisher Art. 251 Vertrag zur Gründung der Europäischen Gemeinschaft (EGV) in der konsolidierten Fassung des Vertrags von Nizza, in: Amtsblatt der EU, Nr. C 325 vom 24. Dezember 2002, S. 33-184.

31 Simon Bulmer/David Dolowitz/Peter Humpreys/Steven Padgett: Policy transfer in European Union governance. Regulating the utilities, Oxon/New York 2007, S. 122-123.

32 Ebenda, S. 123. 
Konkret lassen sich diese konzeptionellen Überlegungen am Beispiel der Einsetzung der ERGEG durch einen Beschluss der Kommission vom 11. November 2003, ${ }^{33}$ wie auch durch die Initiierung des Florenz Forums im Jahr 1998, veranschaulichen. Vor allem das letztgenannte ,governance'-Netzwerk fördert den informellen, transnationalen Dialog zwischen den teilnehmenden Akteuren und die Vereinbarung unverbindlicher Regelungen zur grenzüberschreitenden Marktharmonisierung und -integration. Neben EU-weiter Regelsetzung und nationaler Implementierung stellt der Forumsprozess nach Burkard Eberlein ${ }^{34}$ einen „dritten Weg“ der Energiemarktregulierung dar, der auf dem Prinzip der ,regulation by cooperation“ basiert. Dieses Konzept zeichnet sich dadurch aus, dass die zwischen den beteiligten Akteuren getroffenen Vereinbarungen nicht rechtsverbindlich sind, aber dennoch zu einem späteren Zeitpunkt per Gesetzgebung Rechtsverbindlichkeit erlangen können. Informelle Netzwerke, wie das Florenz Forum, dienen daher unter anderem zur vorbereitenden Konsensbildung zwischen der Europäischen Kommission, Marktakteuren, Regulierungsbehörden und Mitgliedstaaten, um Unterstützung für Gesetzesinitiativen oder für die formale Annahme von informellen Beschlüssen sicherzustellen. ${ }^{35}$ In diesem Zusammenhang kann beispielsweise auf die Verordnung Nr. 1228/2003 über Netzzugangsbedingungen für den grenzüberschreitenden Stromhandel sowie auf Teile des „Dritten Binnenmarktpakets“ hingewiesen werden, da beide eine klare Übereinstimmung mit zuvor erzielten Verhandlungsergebnissen des Florenz Forums erkennen lassen. Die Neuausrichtung der Energiepolitik der Europäischen Union zugunsten eines regionalen Integrationsansatzes weist dieselben Charakteristika auf. Wie im Folgenden dargestellt wird, geht in diesem Fall nicht nur der Ursprung des ,bottom up'-Konzepts auf das Florenz Forum oder genauer gesagt auf eine Initiative der Regulierungsbehörden zurück, sondern dessen Ausgestaltung und Umsetzung erfolgt ebenfalls auf Grundlage informeller , market governance'.

Wie eingangs angedeutet, standen die Themen Marktliberalisierung und Netzregulierung im Zentrum der ersten EU-Strommarktreformen. Das Thema Marktintegration hingegen wurde zu dieser Zeit vor allem im Florenz Forum diskutiert. Die Idee der Einführung regionaler Kooperationsstrukturen als möglichen Zwischenschritt zum Binnenmarkt wurde auf Initiative des „Council of European Energy Regulators (CEER)“36 im Rahmen des 10. Forums im Jahr 2003 erstmals offiziell vorgebracht. Vom Verbund der europäischen Energiebörsen, der „Association of European Power Exchanges (Europex)“, sowie von der damaligen Vereinigung der europäischen Übertragungsnetzbetreiber, „Association of European Transmission System Operators (ETSO)“37, wurde dieser Vorschlag ausdrücklich begrüßt. ${ }^{38}$ Da die Europäische Kommission zur selben Zeit ein neues Strategiepapier zum Energiebin-

33 Beschluss der Kommission vom 11. November 2003 zur Einsetzung der Gruppe der europäischen Regulierungsbehörden für Elektrizität und Erdgas (2003/796/EG), in: Amtsblatt der EU, Nr. L 296 vom 14. November 2003, S. 34-35.

34 Burkard Eberlein: Regulation by Cooperation: The 'Third Way' in making rules for the Internal Energy Market, in: Peter Cameron (Hrsg.): Legal Aspects of EU Energy Regulation. Implementing the new Directives on Electricity and Gas across Europe, Oxford/New York 2005, S. 59-88.

35 Eberlein: The Making of the European Energy Market, 2008, S. 80.

36 Das „Council of European Energy Regulators (CEER)“ repräsentiert einen freiwilligen Zusammenschluss der unabhängigen nationalen Energieregulierungsbehörden mit dem Ziel Verbraucherinteressen zu schützen und die Schaffung eines einheitlichen europäischen Energiebinnenmarktes voranzutreiben.

37 Im Jahr 1999 wurde der Verbund der „European Transmission System Operators“ (ETSO) von den vier regionalen Vereinigungen der Übertragungsnetzbetreiber mit dem Ziel der europaweiten Harmonisierung der Rahmenbedingungen für Netzzugang, Netznutzung und den grenzüberschreitenden Stromhandel gegründet. ETSO wurde im Juli 2009 aufgelöst und alle Aufgaben auf den neuen Verbund „European Network of Transmission System Operators für Electricity (ENTSO-E)“ übertragen.

38 Florenz Forum: Conclusions - Tenth Meeting of the European Electricity Regulatory Forum Rome. 8/9 July 2003. 
nenmarkt vorbereitete, das auch im Florenz Forum zur Sprache kam, wurde der , bottom up “Ansatz regionaler Marktintegration auf Druck der genannten Befürworter schließlich in das Strategiepapier der Kommission einbezogen. Die sogenannte „Medium-term vision for the Internal Electricity Market“ (2004) ${ }^{39}$ ist somit das erste offizielle Dokument der Europäischen Union, das die Schaffung eines gemeinsamen Energiebinnenmarktes über den Zwischenschritt regionaler Energiemärkte anerkennt.

2005, ein Jahr nach Vorlage des Strategiepapiers, präsentierte ERGEG auf dem 12. Florenz Forum ein Konsultationspapier zur Konkretisierung des Konzepts regionaler Energiemärkte und erfuhr im Verlauf des Konsultationsverfahrens große Zustimmung von den beteiligten Interessenvertretern aus Politik und Energiewirtschaft. ${ }^{40}$ Daraufhin initiierte ERGEG im Jahr 2006 ein neues, governance'-Modell, die sogenannten ,Regionalen Initiativen". Diese sahen die Einteilung der 27 europäischen Strommärkte in sieben, sich überlappende Regionalmärkte vor, die, einem klaren Aktionsplan folgend, in den Bereichen Infrastrukturausbau, Engpassmanagement, Regulierung, Markttransparenz sowie bei der Bereitstellung von Regelenergie eine bessere grenzüberschreitende Koordinierung und Harmonisierung anstreben sollten. Jede Region wurde mit einem Koordinationsgremium, dem „Regional Coordination Committee (RCC)“41, einer „Implementation Group (IG)“442 und einer „Stakeholder Group (SG“) ${ }^{43}$ ausgestattet. Zur effektiveren Koordination der einzelnen „Regionalen Initiativen“ wurde zudem eine nationale Regulierungsbehörde pro Region als , lead regulator" eingesetzt und eine ERGEG-Arbeitsgruppe ins Leben gerufen, die die kohärente Umsetzung der Integrationsmaßnahmen in den einzelnen Regionen überwachen soll. ${ }^{44}$

Die dargestellte dezentrale Form der ,market governance' innerhalb der Europäischen Union stellt somit ein Novum im europäischen Integrationsprozess dar. Entgegen der bisherigen Praxis wurde mit den „Regionalen Initiativen“ erstmals ein Experiment vollzogen, das zumindest bis zu seiner Vollendung in gewisser Weise im Widerspruch zum bislang gültigen Paradigma des gemeinsamen Marktes steht. Die Meriten für diesen energiepolitischen Richtungswechsel jedoch allein der Europäischen Kommission zuzuordnen, würde zu kurz greifen. Vielmehr haben drei wesentliche Faktoren diesen pragmatischen Wandel ausgelöst: (1) die bereits existierenden, signifikanten transnationalen Handelsströme zwischen benachbarten Mitgliedstaaten in Teilen der Europäischen Union; (2) der Mangel an Handlungsalternativen; (3) die Gewissheit, dass eine Vereinheitlichung der Marktstrukturen mittels Zentralisierung weder politisch noch wirtschaftlich durchführbar sein würde. Die dargelegte Einrichtung regionaler Kooperationsstrukturen, wie der ERGEG „Regionalen Initiative Strom“, gibt dabei allerdings noch keinen Anlass zu der Vermutung einer Neudefinition energiepolitischer Zielsetzungen der Kommission. Erst die spätere formaljuristische Aufwertung regionaler Zusammenarbeit der oben genannten Akteure durch die Verabschiedung des „Dritten Binnenmarktpakets“ im Juli 2009 manifestiert unzweifelhaft die pragmatische Neuausrichtung der EU-Energiepolitik zugunsten eines zweistufigen Integrationsansatzes.

39 Europäische Kommission: DG Energy and Transport Working Paper. Strategy Paper. Medium term vision for the internal electricity market, Brüssel, 01.03.2004.

40 Florenz Forum: Conclusions - Twelfth Meeting of the European Electricity Regulatory Forum Florence. 1-2 September 2005.

41 Das „Regional Coordination Committee (RCC)“ setzt sich aus den nationalen Regulierungsbehörden der Region zusammen.

42 Der „Implementation Group (IG)“ gehören die Regulierungsbehörden und die zentralen Interessenvertreter der Region an.

43 Die „Stakeholder Group (SG)“ bietet eine Dialogplattform für alle beteiligten Marktakteure einer Region.

44 Informationen zu den „Regionalen Initiativen“ sind abrufbar unter: http://www.energy-regulators.eu/portal/ page/portal/EER_HOME/EER_INITIATIVES/ERI (letzter Zugriff: 30.01.2011). 
Abbildung 1: ERGEG ,Regionale Initiative“ - Strom

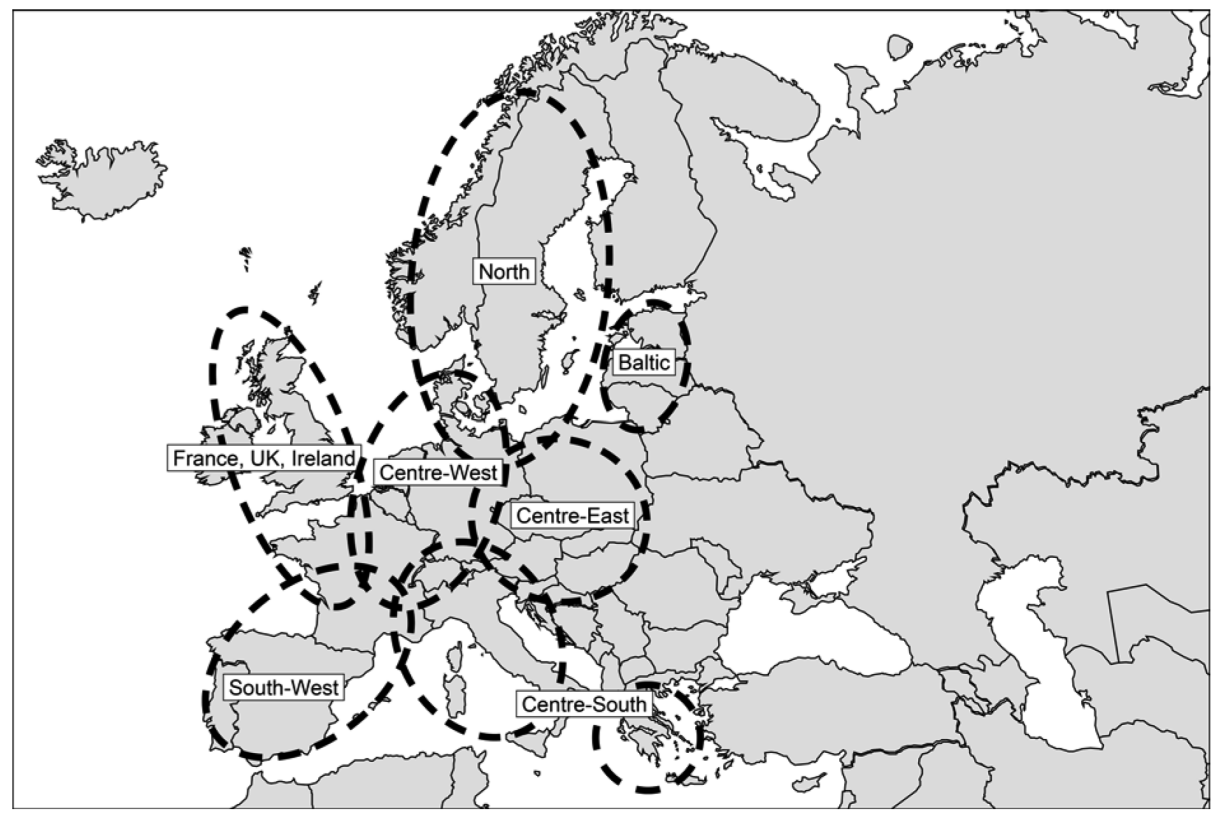

Quelle: Eigene Darstellung auf Basis von Rte - réseau de transport d'electricité.

Die formelle Anerkennung regionaler Kooperation und Integration im europäischen Sekundärrecht erfolgte aber keineswegs am Rande, sondern findet sich an exponierter Stelle sowohl in der Richtlinie 2009/72/EG zum Elektrizitätsbinnenmarkt $t^{45}$ als auch in der novellierten Verordnung Nr. 714/2009 über Netzzugangsbedingungen für den grenzüberschreitenden Stromhandel. ${ }^{46}$ Im erstgenannten Fall heißt es in Art. 6 Abs. 1 unter anderem: „Die Mitgliedstaaten sowie die Regulierungsbehörden arbeiten zusammen, um als ersten Schritt hin zum [sic!] einem vollständig liberalisierten Binnenmarkt ihre nationalen Märkte auf einer oder mehreren regionalen Ebenen zu integrieren." In der genannten Verordnung werden diese allgemeinen Vorschriften, mit dem Hinweis auf die besondere Rolle der Übertragungsnetzbetreiber, weiter präzisiert. Dort in Art. 12 wird diesen deshalb nicht nur die Aufgabe zugeordnet, innerhalb des europäischen Verbunds der Übertragungsnetzbetreiber ENTSO-E die regionale Zusammenarbeit zu stärken, sondern explizit im Hinblick auf ein verbessertes Netzmanagement, die Bereitstellung von Regelenergie und die diskriminierungsfreie Vergabe grenzüberschreitender Übertragungskapazitäten durch regionale Vereinbarungen zu treffen. Ferner werden die Netzbetreiber dazu verpflichtet alle zwei Jahre einen regionalen Investitionsplan aufzustellen, an dem sich der grenzüberschreitende Infrastrukturausbau in der jeweiligen Region orientieren soll.

In Übereinstimmung mit dem von Eberlein ${ }^{47}$ postulierten ,dritten Weg“ der Marktregulierung fällt auf, dass in der Tat auch das Konzept regionaler Integration, zwar auf der Ebene informeller Netzwerke initiiert, nachträglich aber formale Aufwertung durch europäisches Sekundärrecht erfuhr.

45 Richtlinie 2009/72/EG über gemeinsame Vorschriften für den Elektrizitätsbinnenmarkt.

46 Verordnung Nr. 714/2009 über die Netzzugangsbedingungen.

47 Eberlein: Regulation by Cooperation, 2005. 


\section{Der Prozess differenzierter Integration am Beispiel des Energiebinnenmarktes}

Ausgehend von den bisherigen Ausführungen und angesichts des fünfjährigen Bestehens der „Regionalen Initiativen“ erscheint ein Blick auf den gegenwärtigen Zustand der Marktintegration angebracht. Wie im vorigen Abschnitt angedeutet, legt die Wahl eines regionalen Integrationsansatzes die Vermutung nahe, dass einzelne Marktregionen sich in unterschiedlichem Tempo entwickeln. Um dieses Phänomen konzeptionell zu fassen, wird der Strommarkt im Folgenden aus dem Blickwinkel differenzierter Integration ${ }^{48}$ betrachtet. Dieses theoretische Konzept, wie von Alexander $\mathrm{Stubb}^{49}$ kategorisiert, umfasst den Integrationsprozess in drei verschiedenen Ausprägungen: „Time“, „Space“ und „Matter“. Für die vorliegende Analyse wird die zeitliche Dimension des Konzepts genutzt. Diese begreift, differenzierte Integration“ im Sinne einer ,multi-speed EU“50 oder als Europa der verschiedenen Geschwindigkeiten und eignet sich deshalb hervorragend, um die Verschmelzung nationaler Strommärkte zu regionalen Einheiten beschreibbar zu machen. Obwohl es in erster Linie auf politische Integrationsprozesse abzielt, wird das Konzept im vorliegenden Fall auf wirtschaftliche und regulative Integrationsmechanismen in der Europäischen Union angewendet. Die folgenden Ausführungen untersuchen deshalb die zentralen Integrationsfortschritte und -hemmnisse bei der Schaffung regionaler Strommärkte anhand wirtschaftlicher Indikatoren und regulativer Harmonisierung.

Grundsätzlich lassen empirische Indikatoren von Marktintegration, wie grenzüberschreitende Handelsvolumina, Preiskonvergenz, Harmonisierung der Marktregulierung und des Netzmanagements auf den Zustand des jeweiligen geografischen Marktes schließen. Was den physischen Stromhandel in absoluten Zahlen angeht, zeichnet sich insbesondere in Zentral- und Westeuropa der größte grenzüberschreitende Austausch innerhalb der Europäischen Union ab. ${ }^{51}$ Der intra-regionale Handel, gemessen am gesamten Stromhandel in dieser Region, fällt mit circa 10 Prozent noch relativ moderat aus. ${ }^{52}$ Zudem fällt auf, dass die am Rand gelegenen Mitgliedstaaten und Regionen, wie die iberische Halbinsel, Großbritannien und die Baltischen Staaten weniger grenzüberschreitenden Stromhandel mit ihren Nachbarn treiben. Sie stellen bislang relativ isolierte Marktgebiete dar. Die osteuropäischen Märkte sind dagegen schon recht stark am innereuropäischen Handel beteiligt und handeln, gemessen an ihrem regionalen Gesamtverbrauch, bereits mehr als 20 Prozent des Stroms mit ihren westlichen Nachbarn. ${ }^{53}$ Hauptursachen für die geringen grenzüberschreitenden Stromflüsse zwischen Spanien und Frankreich sowie an der britisch-französischen Seegrenze sind die mangelnde Leitungsinfrastruktur und die abweichenden Marktdesigns. Um eine bessere infrastrukturelle Verknüpfung Südwesteuropas mit Frankreich zu gewährleisten, soll eine Leitung durch die Pyrenäen ab 2013 doppelt so große Stromflüsse zwischen Spanien und Frankreich zulassen wie bisher. ${ }^{54}$ Des Weiteren werden gegenwärtig Stromleitungen zwi-

48 Für einen Überblick zur historischen Entwicklung des Konzepts der differenzierten Integration und zum Stand der gegenwärtigen wissenschaftlichen Debatte siehe Rudolf Hrbek: Der Integrationsprozess und das Konzept , differenzierte Integration', in: Udo Diedrichs/Anne Faber/Funda Tekin/Gaby Umbach (Hrsg.): Europe Reloaded. Differentiation or Fusion?, Baden-Baden 2011, S. 78-110.

49 Alexander Stubb: A Categorization of Differentiated Integration, in: Journal of Common Market Studies 2/1996, S. 283-295, hier 286-287.

50 Ebenda, S. 287.

51 ENTSO-E: Statistical Yearbook, Brüssel 2008, S. 137.

52 Europäische Kommission: Interconnecting Europe. New perspectives for Trans-European Energy Networks. Luxembourg 2008.

53 Ebenda.

54 Süddeutsche Zeitung: Lange Leitung zur Wüste. Siemens soll die Stromnetze Frankreichs und Spaniens verbinden, 18./19.12.2010. 
schen Großbritannien und Frankreich erweitert und auch die Anbindung der skandinavischen Länder an Kontinentaleuropa wurde in jüngster Zeit durch neue Leitungsinfrastruktur verbessert.

\section{Abbildung 2: Zusammensetzung der ERGEG ,Regionalen Initiativen“ für den Strom- markt}

\begin{tabular}{|c|l|l|}
\hline \multicolumn{1}{|c|}{ Region } & \multicolumn{1}{|c|}{$\begin{array}{c}\text { Mitglieder von nur einer } \\
\text { Marktregion }\end{array}$} & \multicolumn{1}{|c|}{$\begin{array}{c}\text { Mitglieder von mehreren } \\
\text { Marktregionen }\end{array}$} \\
\hline Baltic & Estland, Lettland, Litauen & \\
\hline Central-East (CE) & $\begin{array}{l}\text { Tschechische Republik, Ungarn, } \\
\text { Slowakei }\end{array}$ & $\begin{array}{l}\text { Österreich, Deutschland, Polen, } \\
\text { Slowenien }\end{array}$ \\
\hline Central-South (CS) & Griechenland, Italien & $\begin{array}{l}\text { Österreich, Frankreich, } \\
\text { Deutschland, Slowenien }\end{array}$ \\
\hline Central-West (CW) & $\begin{array}{l}\text { Belgien, Luxemburg, } \\
\text { Niederlande }\end{array}$ & Frankreich, Deutschland \\
\hline Northern & $\begin{array}{l}\text { Dänemark, Finnland, } \\
\text { Norwegen, Schweden }\end{array}$ & Deutschland, Polen \\
\hline South-West (SW) & Portugal, Spanien & Frankreich \\
\hline France-UK-Ireland (FUI) & Irland, Großbritannien & Frankreich \\
\hline
\end{tabular}

Quelle: Eigene Darstellung.

Neben der physischen Verknüpfung der einzelnen Regionen und Mitgliedstaaten spielt jedoch auch die kommerzielle Verflechtung der nationalen Märkte eine zentrale Rolle bei der Marktintegration. Nur wenn Strom und Durchleitungskapazitäten auch grenzüberschreitend gehandelt werden können, entsteht ein wahrhaft integrierter Markt. In dieser Hinsicht übernehmen einige Regionen innerhalb der Europäischen Union eine Vorreiterfunktion. Der am weitesten fortgeschrittene Regionalmarkt, ,Nord Pool“, 55 besteht aus den nordeuropäischen Ländern Schweden, Finnland, Dänemark und dem EU-nicht-Mitglied Norwegen und existiert bereits seit 1996. Er wurde auf Initiative Norwegens und Schwedens gegründet und über die Jahre erweitert. „Nord Pool“ stellt damit bereits seit mehreren Jahren einen integrierten Markt dar, der auf der engen Kooperation zwischen den vier Übertragungsnetzbetreibern und der gemeinsamen Strombörse „Nord Pool Spot“ basiert. ${ }^{56}$

Andere Regionen haben im Zuge der „Regionalen Initiativen“ einen vergleichbaren Pfad eingeschlagen und entwickeln sich mehr und mehr zu integrierten Teilmärkten. Marktkopplungsinitiativen ${ }^{57}$ in den Regionen Zentralwesteuropa (CW-Region) und Nordeuropa (Northern-Region) belegen dies. Das ,trilateral market coupling“ zwischen Frankreich, Belgien und den Niederlanden im Jahr 2006 stellte einen ersten Schritt auf dem Weg hin zu einem integrierten Strommarkt in Kontinentaleuropa dar. Die Kopplung der drei Märkte äußert sich

55 In Abbildung 2 wird der skandinavische Großhandelsstrommarkt „Nord Pool” unter der Region „Northern“ geführt, da im Rahmen der Regionalen Initiativen Deutschland und Polen diesem Regionalmarkt ebenfalls zugeordnet wurden.

56 ERGEG: The Creation of Regional Electricity Markets. An ERGEG Conclusions Paper, 2006, Dok. E05-ERF03-06a.

57 Marktkopplung beschreibt die Kooperation von Netzbetreibern und Energiebörsen mit dem Ziel sowohl Strom als auch grenzüberschreitenden Leitungskapazitäten zwischen den involvierten Ländern nach einer integrierten Methode zu handeln beziehungsweise zu auktionieren. Vgl. ENTSO-E: Annual Report 2009, Brüssel, S. 36. 
in konvergierenden oder identischen Preisen, ${ }^{58}$ allerdings nur solange genügend Durchleitungskapazität an den Grenzen der Länder vorhanden ist, um das gehandelte Stromvolumen auch termingerecht zu transportieren. Andernfalls wird das gemeinsame Marktgebiet entweder in zwei oder drei Preiszonen aufgeteilt, um die Netzengpässe durch ein Ausbalancieren von Angebot und Nachfrage wieder zu beseitigen. Anders als bei „Nordpool“ existieren in den drei Ländern trotz der Marktkopplung weiterhin die nationalen Energiebörsen „Powernext“", „Belpex“" und „APX-ENDEX“.

Im Rahmen der regionalen Zusammenarbeit in Zentralwesteuropa haben sich Deutschland und Luxemburg ferner dazu entschlossen eine Preiskopplung mit den bereits gekoppelten Märkten Frankreichs, Belgiens und den Niederlanden durchzuführen und somit einen integrierten Markt in der CW-Region für den kurzfristigen Stromhandel zu schaffen. Parallel zur Vollendung dieses Projekts, das sich derzeit in der Umsetzungsphase befindet, streben der nordeuropäische und später der iberische Markt eine interregionale Marktkopplung mit der CW-Region an. ${ }^{59}$ Gleichzeitig vollzieht sich derzeit bereits die interregionale Marktkopplung zwischen der CW-Region auf der einen Seite und den nordeuropäischen Ländern auf der anderen.

Darüber hinaus haben die Netzbetreiber aus der CW-Region im Jahr 2008 eine gemeinsame Tochtergesellschaft, die „Capacity Allocation Service Company (CASC)“ gegründet, die seitdem als Handelsplattform für den langfristigen grenzüberschreitenden Stromhandel dient. ${ }^{60}$ Auf der Basis einheitlicher regionaler Auktionsregeln wurde so in Fragen des Marktdesigns die Voraussetzung für einen reibungslos funktionierenden Regionalmarkt geschaffen. Die gemeinsame Plattform soll in Zukunft auch den Handel des zentralsüdeuropäischen Marktes (CS-Region) (und der Schweiz) übernehmen und setzt damit harmonisierte Regeln für den langfristigen Stromhandel dieser beiden Regionen.

Während in den genannten Regionen deutliche Integrationsfortschritte beobachtet werden können, verläuft die Harmonisierung und Verknüpfung nationaler Märkte in anderen Regionen weitaus langsamer. Zwar hat seit diesem Jahr auch in Zentralosteuropa (CE-Region) eine gemeinsame Auktionsplattform für den langfristigen regionalen Stromhandel ihre Arbeit aufgenommen, eine Marktkopplung des italienischen und slowenischen Marktes wurde Ende 2010 vollendet und eine Anbindung Großbritanniens an den CW-Markt ist für die kommenden beiden Jahre geplant. Dennoch haben unterschiedliche infrastrukturelle Voraussetzungen sowie inkompatible Marktdesigns gerade in Süd- und Osteuropa einen langsameren Integrationsprozess zur Folge. Heute befinden sich diese Regionen auf einem guten Weg hin zu mehr Harmonisierung und Marktintegration, die Vorreitermärkte im Norden und Westen der Europäischen Union konnten die besseren Ausgangsbedingungen jedoch nutzen und weisen dementsprechend bis dato ein höheres Integrationstempo auf.

Die genannten Beispiele verdeutlichen, dass Marktintegration und -harmonisierung auf Basis regionaler Kooperation der beteiligten Marktakteure, vor allem der Strombörsen und Netzbetreiber, erfolgreiche Integrationsprozesse in Gang setzen kann. Insbesondere der CW-Markt hat dabei gezeigt, wie innerhalb weniger Jahre eine dynamische Verschmelzung nationaler Märkte zu einem Regionalmarkt gelingen kann. Zugegebenermaßen ist in dieser

58 Im Jahr 2008 waren die Preise für den kurzfristigen Stromhandel (in diesem Fall für den nächsten Tag) in 84 Prozent der Zeit identisch. Vgl. ERGEG: Status Review of the Liberalisation and Implementation of the Energy Regulatory Framework. Brussels, 10 December 2009, S. 18.

59 ERGEG: Status Review on the ERGEG Regional Initiatives 2010, Dok. E10-RIG-11-03, S. 29; ERGEG: Safeguarding the move to a single EU Energy market. ERGEG Regional Initiatives Progress Report 2009, S. 32.

60 Everis/Mercados: From Regional Markets to a Single European Market. Final Report, 2010, S. 32; ERGEG: Status Review of the ERGEG Regional Initiatives, 2010, S. 32. 
Region auch die politische Rückendeckung für die Entwicklung regionaler Marktstrukturen am größten, wie die regelmäßigen Konsultationen im Rahmen des „Pentalateral Energy Forum"61 verdeutlichen. Auch der iberische Markt hat sich trotz mangelnder Anbindung an Frankreich weiter integriert und in Osteuropa bildet sich ebenfalls schrittweise ein regionaler Strommarkt heraus. Die geplante Kopplung der CW-Region mit den nordeuropäischen Märkten zeigt ferner, dass regionale Integration keineswegs in eine Sackgasse führt, die eine Zersplitterung des europäischen Marktes zur Folge hat, sondern im Gegenteil den Ausgangspunkt für überregionale Integration bildet und somit Europa dem Ziel eines nahtlos funktionierenden Binnenmarktes näher bringt.

Neben der infrastrukturellen Verknüpfung nationaler Märkte und der Interoperabilität der Marktdesigns sind für die Marktintegration Fragen der grenzüberschreitenden Regulierung, insbesondere der Markttransparenz, von großer Bedeutung. Markttransparenz ist essenziell für einen funktionierenden, integrierten Wettbewerbsmarkt, da nur eine diskriminierungsfreie Bereitstellung von Informationen seitens der Netzbetreiber über aktuelle Stromflüsse, Netzauslastung, Stromerzeugung und verfügbare Übertragungskapazitäten an alle Marktakteure und Regulierungsbehörden einen fairen, effizienten Wettbewerb erlauben. ${ }^{62}$ Das bestehende Sekundärrecht gibt dafür zwar grobe Vorgaben, allerdings lassen diese viel Spielraum zur nationalen Interpretation und bedürfen der Konkretisierung. Zu diesem Zweck hat ERGEG die regionalen Akteure dazu aufgefordert, hinreichend präzise, gemeinsame Transparenzstandards für den regionalen Stromhandel zu formulieren. Verglichen mit den Fortschritten bei der Marktkopplung hat sich in diesem Bereich in den vergangenen Jahren eine wesentlich gleichmäßigere Entwicklung zwischen den Regionen abgezeichnet. Bislang haben sechs von sieben Regionen einen regionalen Markttransparenzbericht vorgelegt, der spezifische Vorgaben zu den genannten Bereichen enthält und die regelmäßige Veröffentlichung von Monitoring-Berichten vorsieht. Trotz fortbestehender Abweichungen zwischen formalen Vorgaben und praktischer Informationsbereitstellung durch die Netzbetreiber, hat ERGEG in seinem letztjährigen Fortschrittsbericht zu den „Regionalen Initiativen“ festgestellt, dass alle in den Regionen eingeführten Transparenzregeln miteinander kompatibel seien und in keinster Weise der Schaffung eines gemeinsamen Marktes im Wege stünden. ${ }^{63}$

Neben der Frage der Transparenz umfasst grenzüberschreitende Marktregulierung auch die Kompetenzausstattung der nationalen Regulierungsbehörden, nationale Verwaltungsprozesse sowie gemeinsame Ansätze zur Marktüberwachung. In dieser Hinsicht wurden in zwei Regionen (Südwesteuropa und Zentralosteuropa) Harmonisierungsbemühungen initiiert und in der Region Zentralwesteuropa die Durchführung eines gemeinsamen ,market monitoring ‘ beschlossen. ${ }^{64}$ Als einzige Region hat Zentralosteuropa, im Einklang mit den Vorgaben des „Dritten Binnenmarktpakets“, bereits einen regionalen Investitionsplan veröffentlicht. In Zentralwesteuropa liegt ein solcher Plan zum gegenwärtigen Zeitpunkt noch nicht vor, eine gemeinsame Methodologie für die Planung und die Investition in Infrastruktur wurde aber vereinbart.

61 Memorandum of Undertstanding of the Pentalateral Energy Forum on Market coupling and security of supply in central western Europe, unterzeichnet am 6. Juni 2007 von den Regierungen Belgiens, Deutschlands, Frankreichs, Luxemburgs und der Niederlande sowie durch die Netzbetreiber, Strombörsen und andere Marktteilnehmer der CW-Region.

62 ERGEG: Progress Report, 2009; ERGEG: Status Review of the ERGEG Regional Initiatives, 2010.

63 Ebenda, S. 34.

64 Everis/Mercados: From Regional Markets to a Single European Market, 2010, S. 50. 


\section{Die EU-Energiestrategie 2020 und die zukünftige Rolle der ,Regionalen Initiativen“}

Mit Blick auf das Konzept der differenzierten Integration konnten einige empirische Belege für die aufgestellte Hypothese variierender Integrationstempi der Strommarktregionen präsentiert werden. Insbesondere beim kommerziellen Stromhandel nehmen zwei Energieregionen, Zentralwesteuropa und Nordeuropa, eine Vorreiterfunktion ein. Die eingeleitete Marktkopplung zwischen beiden Regionen sowie die geplante Ausdehnung dieses Projekts auf andere Regionalmärkte verdeutlicht überdies, dass regionale Integration keineswegs zur Zersplitterung des Binnenmarktes in eine Gruppe von isolierten Teilmärkten führt, sondern die Europäische Union über den Pfad der interregionalen Marktintegration der Vollendung des Energiebinnenmarktes einen Schritt näher bringt. Es stellt sich indes die Frage: Wie und wann der zweite Schritt zum gemeinsamen Energiemarkt eingeleitet wird?

Die im November 2010 von der Kommission vorgelegte Energiestrategie ${ }^{65}$ gibt darüber keine konkrete Auskunft, jedoch identifiziert sie die Schaffung eines ,wahrhaft europaweit integrierten Energiemarkt[es] “ als einen von fünf Schwerpunkten. Neben den Themen Energieeffizienz, Verbraucherrechte, Energietechnologie und Energieaußenpolitik soll die Marktintegration, nach Meinung der Kommission im Wesentlichen durch eine bessere Kopplung der nationalen Stromnetze erreicht werden. Die Beschleunigung von Genehmigungsverfahren, die Vereinfachung von Marktregeln zum Infrastrukturausbau ebenso wie die Schaffung eines angemessenen Finanzierungsrahmens für den Netzausbau stehen hierbei im Mittelpunkt. Auch wenn der spezifischen Rolle regionaler Akteure in der neuen Energiestrategie wenig Aufmerksamkeit geschenkt wird, geht die Kommission in ihrem Infrastrukturpaket $^{66}$ für den Zeitraum bis 2020 und danach explizit auf regionale Cluster als Instrument zur Beschleunigung des Infrastrukturausbaus ein. Eine Neuerung im Zuge des Infrastrukturausbaus dürften in Zukunft projektspezifische regionale Ad-hoc-Kooperationsstrukturen bilden, die parallel zu den von ERGEG etablierten Strommarktregionen einzelne Infrastrukturvorhaben koordinieren. Trotz der erzielten Fortschritte in den einzelnen Marktregionen bleibt die vollständige Umsetzung des Acquis aber auch zukünftig eine Aufgabe der „Regionalen Initiativen“. Es ist außerdem zu erwarten, dass die im „Dritten Binnenmarktpaket" vorgesehene Ausarbeitung von Netzkodizes ${ }^{67}$ durch ENTSO einen weiteren Schwerpunkt für die Arbeit innerhalb der Strommarktregionen bieten wird. Die auf Grundlage von Verordnung Nr. 713/2009 zum 3. März 2011 neu geschaffene „Agentur für die Zusammenarbeit der Energieregulierungsbehörden (ACER)“ wird von nun an die Verantwortung für die kohärente Entwicklung der sieben Marktregionen übernehmen. Sie setzt sich aus den Leitern der 27 nationalen Energieregulierungsbehörden zusammen, übernimmt die vormaligen Tätigkeiten von ERGEG sowie weitere neu geschaffene Aufgaben. Anders als seine Vorgängerorganisation hat ACER jedoch im Rahmen des „Dritten Binnenmarktpakets" den Status einer EU-Institution mit offiziellem Sitz in Ljubljana erhalten. Dabei wird sie vor allem die einheitliche Interpretation der Marktregeln sowie die der Netzkodizes

65 Europäische Kommission: Mitteilung der Kommission an das Europäische Parlament, den Rat, den Europäischen Wirtschafts- und Sozialausschuss und den Ausschuss der Regionen. Energie 2020 - Eine Strategie für wettbewerbsfähige, nachhaltige und sichere Energie, KOM (2010) 639.

66 Europäische Kommission: Mitteilung der Kommission an das Europäische Parlament, den Rat, den Europäischen Wirtschafts- und Sozialausschuss und den Ausschuss der Regionen. Energieinfrastrukturprioritäten bis 2020 und danach - ein Konzept für ein integriertes europäisches Energienetz, KOM (2010) 667.

67 Netzkodizes sind Regeln für den Netzbetrieb, die durch einen Beschluss im Komitologieverfahren Rechtsverbindlichkeit erlangen können und durch das „Dritte Binnenmarktpaket“ eingeführt wurden. Sie betreffen die Bereitstellung und Handhabung des konkreten und transparenten Netzzugangs sowie die zukunftsorientierte Planung und technische Weiterentwicklung der Stromnetze durch die Übertragungsnetzbetreiber. Vgl. Verordnung Nr. 714/2009 über die Netzzugangsbedingungen. 
durch die regionalen Akteure überwachen müssen, um das von der Kommission ${ }^{68}$ angestrebte Ziel einer vollständigen europaweiten Marktkopplung bis zum Jahr 2015 zu erreichen.

\section{Sozialisationsprozesse im Europäischen Parlament}

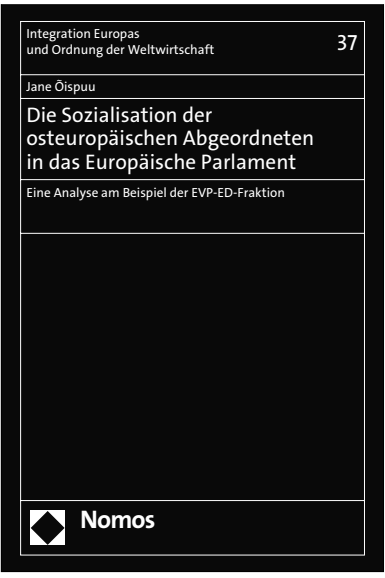

Bitte bestellen Sie im Buchhandel oder versandkostenfrei unter $\bullet$ www.nomos-shop.de

\section{Die Sozialisation der osteuropäischen Abgeordneten in das Europäische Parlament}

Eine Analyse am Beispiel der EVP-ED-Fraktion Von Dr. Jane Oispuu

2011, 302 S., geb., 79,-€

ISBN 978-3-8329-6220-3

(Integration Europas und Ordnung

der Weltwirtschaft, Bd. 37)

nomos-shop.de/13182

Mit der Osterweiterung der Europäischen Union trat eine große Zahl von neuen Akteuren in die europäischen Institutionen ein. Viele Parlamentarier aus den neuen Mitgliedstaaten mussten in das Europäische Parlament integriert werden. Der Band untersucht, wie das Parlament diese Integration so effektiv meistern konnte und wie die neuen Mitglieder ihren Weg in die Parteienverbände fanden.

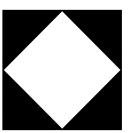

Nomos

68 Europäische Kommission: Mitteilung der Kommission an das Europäische Parlament und den Rat. Die künftige Rolle regionaler Initiativen, KOM (2010) 721. 